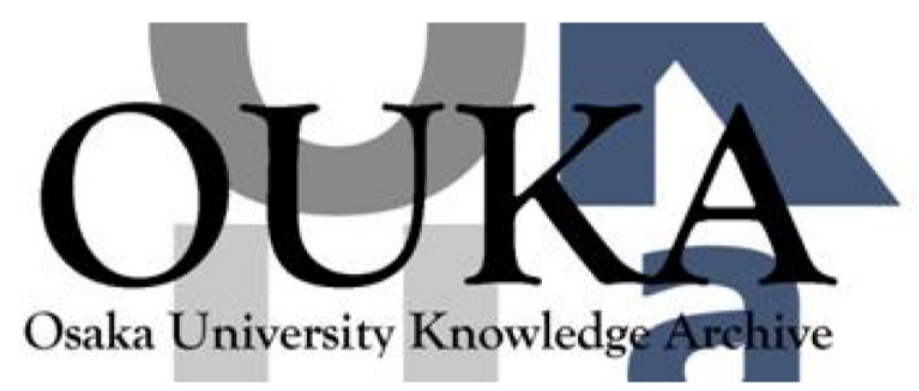

\begin{tabular}{|c|l|}
\hline Title & $\begin{array}{l}\text { Calculation of } \lambda \text {-Transition Temperature in } \\
\text { Liquid } \wedge \text { } 4 \text { He }\end{array}$ \\
\hline Author(s) & Sasaki, Shosuke \\
\hline Citation & Physica B: Condensed Matter. 329(1) p. 232-p. 233 \\
\hline Issue Date & $2003-05$ \\
\hline oaire:version & AM \\
\hline URL & https://hdl.handle.net/11094/27148 \\
\hline rights & $\begin{array}{l}\text { Copyright } 02003 \text { Elsevier Science B. V. All } \\
\text { rights reserved. }\end{array}$ \\
\hline Note & \\
\hline
\end{tabular}

Osaka University Knowledge Archive : OUKA

https://ir. Library. osaka-u. ac. jp/

Osaka University 


\title{
Calculation of $\lambda$-Transition Temperature in Liquid ${ }^{4} \mathrm{He}$
}

\author{
Shosuke Sasaki ${ }^{\text {a,1 }}$ \\ ${ }^{a}$ Shizuoka Institute of Science and Technology, Toyosawa 2200-2, Fukuroi 437-8555, Japan
}

\begin{abstract}
In experiments of Liquid ${ }^{4} \mathrm{He}$, the elementary excitation energy varies with changing of the temperature. This is caused by the non-linear dependence of the total energy upon the number-distribution of elementary excitations. Taking this effect into consideration, we can numerically calculate the $\lambda$ transition temperature. The results are $2.18 \mathrm{~K}$ for the saturated vapor pressure. Other calculated values are $2.8 \mathrm{~K}$ after Landau, and $3.16 \mathrm{~K}$ after London. Since the experimental value is $2.172 \mathrm{~K}$, our calculated value is well in accord with the experimental data.
\end{abstract}

Key words: lambda transition; superfluidity; helium4; phase diagram

\section{Introduction and total energy of liquid ${ }^{4} \mathrm{He}$}

The values of the $\lambda$ transition temperature have been theoretically calculated by two methods until now. F. London has obtained the temperature by neglecting interactions between ${ }^{4} \mathrm{He}$ atoms. His result is $3.16 \mathrm{~K}$ for saturated vapor pressure [1]. Landau and Khalatnikov have calculated the temperature on the basis of Landau theory, and have got $2.8 \mathrm{~K}$ [2]. Its experimental value is $2.172 \mathrm{~K}$ [3]. There is a large difference between the theoretical values and the experimental value, and so we need to find another theory deriving a good value of the $\lambda$ transition temperature. In this paper, we obtain new theoretical values for various pressure values.

A total energy of any many-body-system should be the sum of the kinetic energy of the center of mass and Galilean-invariant terms. So, any three-dimensionalsystem should have a general form of a total energy as

$E=\sum_{p} \frac{\mathbf{p}^{2}}{2 m} n_{p}+\sum_{p, q} \frac{1}{V} f(\mathbf{p}-\mathbf{q}) n_{p} n_{q}+\cdots$.

where $n_{q}$ is the number of the dressed-bosons with momentum $q$. All the dressed-bosons have zero mo-

\footnotetext{
1 Corresponding author. Present address: Shizuoka Institute of Science and Technology, Toyosawa 2200-2, Fukuroi 437-8555, Japan E-mail: sasaki@ns.sist.ac.jp
}

mentum in the ground-state of liquid helium, as is shown in Ref.[4]. The elementary excitation energy $\epsilon_{p}$ at zero temperature is the energy increment from the state $\left\{n_{0}=N\right\}$ to the state $\left\{n_{0}=N-1, n_{p}=\right.$ $1\}$. This functional form $\epsilon_{p}$ determines the functional forms $f, \cdots$ in Eq.(1), consequently the total energy becomes

$$
\sum_{p} \frac{\mathbf{p}^{2}}{2 m} n_{p}+\frac{0.5}{N-1} \sum_{p, q}\left(\epsilon_{p-q}-\frac{(\mathbf{p}-\mathbf{q})^{2}}{2 m}\right) n_{p} n_{q}+E_{\mathrm{G}} .
$$

This gives $E=E_{\mathrm{G}}$ for $\left\{n_{0}=N\right\}$, and $E=\epsilon_{p}+E_{\mathrm{G}}$ for $\left\{n_{0}=N-1, n_{p}=1\right\}$. From subtraction of these two results, the energy increment by one excitation becomes $\epsilon_{p}$ certainly.

In an ultra low temperature, the total energy becomes $E \simeq \sum_{p} \epsilon_{p} n_{p}+E_{\mathrm{G}}$ because $\frac{n_{0}}{N} \simeq 1$. This form is identical with the sum of the excitation energy in Landau theory and the ground state energy $E_{\mathrm{G}}$.

For any temperature $T$ smaller than the $\lambda$ transition temperature $T_{\lambda}$, the number distribution $\left\{n_{p}\right\}$ of the dressed-bosons is derived from the solution of the following equations as

$n_{p}=\left(\exp \left(\frac{\omega_{p}-\mu}{k_{\mathrm{B}} T}\right)-1\right)^{-1}$.

where $\mu$ is the chemical potential, and 
$\omega_{p}=\frac{\partial E}{\partial n_{p}}, \mu=\omega_{0}=\frac{\partial E}{\partial n_{0}}, n_{0}=N-\sum_{p \neq 0} n_{p}$.

Production of one elementary excitation is the same phenomenon that one dressed-boson with momentum 0 is annihilated and one dressed-boson with momentum $p$ is created. Therefore, the elementary excitation energy at any temperature $T$ is given by $\epsilon(p, T)=$ $\omega_{p}-\omega_{0}$. Consequently, the theoretical excitation energy depends upon the temperature. For a small momentum $p$, the equations (2)-(4) have the solution as

$\epsilon(p, T)=\epsilon_{p} \frac{n_{0}}{N}+\frac{p^{2}}{2 m}\left(1-\frac{n_{0}}{N}\right)+$ higher order.

We don't succeed yet to find the functional form of the elementary excitation energy near the roton minimum. Therefore, we use the experimental data of Dietrich, Graf, Huang, and Passell [5] instead of the theoretical values near the roton minimum, as follows;

$\epsilon(p, T)=\Delta(T)+(p-Q)^{2} /\left(2 m_{\text {roton }}\right)$.

At the $\lambda$ transition temperature, these three parameters $\Delta_{\lambda}, Q_{\lambda}, m_{\text {roton }}$ depend on the pressure as $\Delta_{\lambda}=$ $0.409-0.0067 P, Q_{\lambda} / \hbar=1.925+0.0045 P, m_{\text {roton }}=$ $(0.117-0.00014 P) m$, where $P$ is the value of (pressure/bar), the unit of $\Delta_{\lambda}$ is $\mathrm{meV}$, and the unit of $Q_{\lambda} / \hbar$ is inverse of angstrom. We illustrate the excitation energy near the transition temperature in Fig.1.

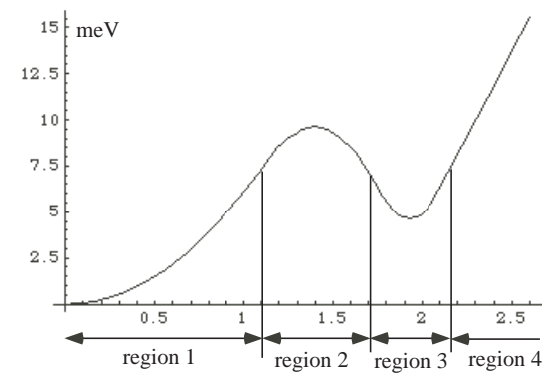

Fig. 1. Excitation energy at $\lambda$ point for the pressure of 1 bar.

Here, we have used Eq.(5), and (6) for the momentum region 1and 3, respectively. For the region 4, we also use the experimental data of Dietrich et al. The result is $\epsilon\left(p, T_{\lambda}\right)=d_{\lambda} p+e_{\lambda}$ where $d_{\lambda}=238.6+6.19 P-$ $0.077 P^{2}$. In the remaining region 2 , there is no detail data near the $\lambda$ point, and therefore we use the function as

$\epsilon\left(p, T_{\lambda}\right)=a_{\lambda} p^{2}+b_{\lambda} p+c_{\lambda}$.

where the values of $a_{\lambda}, b_{\lambda}, c_{\lambda}$ are determined by the following three conditions. (1) The function of Eq.(7) should be continuously connected to the function of Eq.(6) at the boundary between the regions 2 and

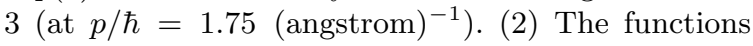

of Eqs.(7) and (6) have the common tangent at this boundary. (3) The functions of Eq. (7) and (5) have the common tangent at the boundary between the regions 1 and 2. Thus all the values of the coefficients have been determined, and we have no free parameter in the function described the elementary excitaion energy.

\section{2. $\lambda$ transition temperature and Conclusion}

We substitute the distribution function Eq.(3) into Eq.(4), and obtain

$\frac{n_{0}}{V}=\frac{N}{V}-\frac{1}{V} \sum_{p \neq 0}\left(\exp \left(\frac{\epsilon(p, T)}{k_{\mathrm{B}} T}\right)-1\right)^{-1}$.

Using Eq.(8), we determine the $\lambda$ transition temperature $T_{\lambda}$ at which the value of $n_{0} / V$ becomes zero. The results are shown in Fig.2.

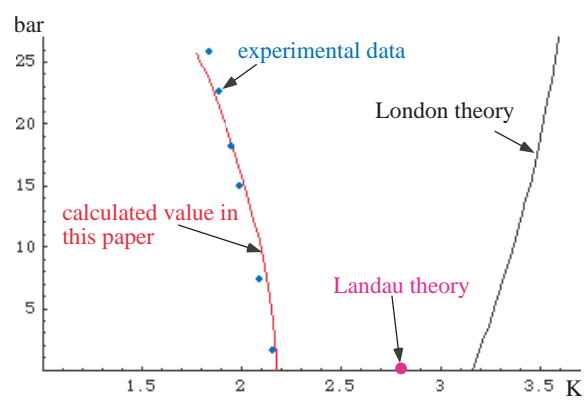

Fig. 2. Theoretical and experimental values of $T_{\lambda}$.

As is seen in Fig.2, the present results are well in accord with the experimental data for all pressure regions. For the saturated vapor pressure, the theoretical value of Landau is $2.8 \mathrm{~K}$, and one of London is $3.16 \mathrm{~K}$. The present result is $2.18 \mathrm{~K}$, and the experimental value is $2.172 \mathrm{~K}$. Thus, the present theory is in good agreement with the experimental result. In the present theory, the calculated $\lambda$ transition temperature decreases for increasing of pressure, this behavior is in accord with the experimental data.

\section{References}

[1] F. London, Superfluid Vol.2, (Dover Publications, 1964) 44.

[2] L. D. Landau and E. M. Lifshitz, Statistical Physics, 2nd Edition (Pergamon Press 1968) 195.

[3] G. Ahlers, Phys. Rev. A8 (1973) 530.

[4] S. Sasaki, Physica B 165 (1990) 507; Springer Series in Solid-State Sciences Vol.79 (Springer 1989) 160; JJAP Suppl. Proc. LT18 26-3 (1987) 23.

[5] O.W.Dietrich, E.H.Graf, C.H.Huang, and L.Passell, Phys. Rev. A 5 (1972) 1377. 